\title{
A DOA Estimation Algorithm from Sparse Representation Perspective for Vehicular Application
}

\author{
Beirui ZHANG ${ }^{1, a}$, Xiaoyou $\mathrm{YU}^{1, b^{*}}$, Heng $\mathrm{YE}^{1}$, Yan $\mathrm{ZHOU}^{1}$, Nengda $\mathrm{Cl}^{1}$, \\ Pengshuai SUN ${ }^{1}$, Gaobo YANG $^{1}$ \\ ${ }^{1}$ Department of Communication Engineering, School of Information Science and Engineering, \\ Hunan University, Changsha, 410082, China \\ aemail: brriot@162.com, bemail: yu.xiaoyou@163.com
}

Keywords: Direction-of-Arrival Estimation; Sparse Representation; Vehicle Communication

\begin{abstract}
In this paper, a recovery algorithm of direction-of-arrival (DOA) Estimation based on weighted $l_{1}$ minimization for vehicular applications is proposed. We construct an overcomplete basis based on the largest eigenvector of the covariance matrix, aiming at a sharp spatial spectrum that exhibits the high-precision. The theoretical analysis and simulation results demonstrate that the proposed method has an excellent performance in the aspects of accuracy.
\end{abstract}

\section{Introduction}

With the rapid development of science and technology, vehicle communication system has aroused people's attention [1][2]. As an important member of the system, cars can realize the interaction of information as well as the intelligent perception of the surrounding environment [3][4]. In order to get the azimuth of targets, DOA estimation plays an important role.

There has existed a number of high resolution algorithms for DOA estimation. The subspace algorithms need a prior knowledge of source number, and relay on a large number of sampling data, or a high signal-to-noise ratio(SNR)[5]. In recent years, Donoho et al proposed the theory of compressed sensing [6], which provides a new idea for DOA estimation. In [7], Malioutov proposed $l_{1}$-SVD, which combines the SVD step of the subspace algorithms with a sparse recovery method based on $l_{1}$ minimization. $l_{1}$-SVD algorithm achieves high resolution when the number of snapshots is small and the sources is correlated. In [8], according to the covariance matrix satisfy the asymptotic Gauss distribution, Stoica proposed SPICE algorithm. Based on [7], Yin proposed $l_{1}$-SRACV algorithm to avoid the selection of regularization parameter [9]. To enforce sparsity, the literature [10] proposes a weighted $l_{1}$ minimization using the property of noise subspace. In [11], a weighted sparse representation model is proposed by applying Capon spectrum. However these methods need a prior knowledge of source number in order to ensure correct division between signal subspace and noise subspace or reduce the dimension of the received signal matrix.

In this paper we presents a weighted $l_{1}$ minimization method for DOA estimation. The method utilizes the largest eigenvector instead of the signal subspace as a sparse vector . And we design a weighted $l_{1}$ norm matrix whose weights correspond to the largest eigenvector to obtain a better approximation of $l_{0}$ norm. Simulations are presented to prove the proposed algorithm effectively suppress the pseudo peaks and has higher estimation accuracy and resolution.

\section{Application Scenario and Sparse Representation Model}

In vehicle communication system, vehicles can achieve information interaction. Furthermore, vehicles need to perceive the location of surrounding vehicles. Fig 1 depicts an application scenario in intelligent transportation system. Vehicle A transmits signal to the target vehicle B in the road. At the same time, A receives the echo signal reflected by the target vehicle. The direction of the echo signal indicates the location of vehicle B. 




Fig 1 Vehicle communication application scenario

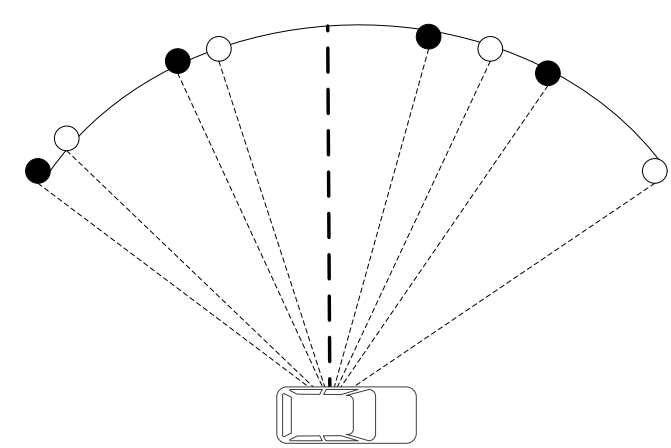

Fig 2 The sparse representation of DOA

At the receiver, sensor arrays can receive the signal from all directions. We pose DOA estimation as a sparse signal representation problem, where we construct a sparse spatial spectrum. As shown in Fig 2, the solid point represents the real source, and the hollow point is the virtual source. And the number of virtual source is greater than the real source. Then this problem can cast as an overcomplete representation in terms of all possible source locations.

\section{DOA Estimation Based on Weighted $l_{1}$ Minimization}

Consider a uniform linear array of $M$ sensors receiving $K$ narrowband signals coming from directions $\left\{\theta_{1}, \theta_{2}, \ldots, \theta_{K}\right\}$. The received signal of the array is expressed as

$$
\mathbf{Y}=\mathbf{A S}+\mathbf{N}
$$

where $\mathbf{Y}$ is $M \times L$ dimensional received signal vector, $\mathbf{S}$ is $K \times L$ dimensional source vector and $\mathbf{N}$ is $M \times L$ dimensional additive Gaussian white noise vector. $L$ is the number of snapshots taken from the array. The matrix $\mathbf{A}$ is the array steering matrix given by $\mathbf{A}=\left[\boldsymbol{\alpha}\left(\theta_{1}\right), \boldsymbol{\alpha}\left(\theta_{2}\right), \ldots, \boldsymbol{\alpha}\left(\theta_{K}\right)\right]$ with the steering vector $\boldsymbol{\alpha}\left(\theta_{k}\right)=\left[1, e^{-j 2 \pi d \sin \theta_{k} / \lambda}, \ldots, e^{-j 2 \pi(M-1) d \sin \theta_{k} / \lambda}\right]^{T}$, where [* $]^{T}$ denotes transpose.

Without loss of generality, the signal and the noise are assumed to be uncorrelated, then the sampling covariance matrix of the array data is given by

$$
\mathbf{R}=\frac{1}{L} \mathbf{Y} \mathbf{Y}^{H}
$$

where $H$ denotes Hermitian transpose. After eigenvalue decomposition of $\mathbf{R}$, we can get the signal subspace matrix $\mathbf{U}_{\mathrm{S}}$ and the noise subspace matrix $\mathbf{U}_{\mathbf{N}}$. Since the signal subspace matrix spans the same space of the array steering matrix, i.e. $\operatorname{span}\left(\mathbf{U}_{\mathbf{S}}\right)=\operatorname{span}(\mathbf{A})$. Suppose that there is a full rank matrix $\mathbf{C}$ such that $\mathbf{U}_{\mathbf{S}}=\mathbf{A C}$, which means that the signal subspace is a linear transform of the steering vector matrix. So the largest eigenvector $\mathbf{e}_{\max }$ also can be expressed as

$$
\mathbf{e}_{\max }=\sum_{k=1}^{K} c_{k} \boldsymbol{\alpha}\left(\theta_{k}\right)
$$

where $c_{k}, k=1,2, \ldots, K$ is a linear combination factor.

Under the sparse representation framework, we divide the whole detection area of interest into some discrete grid of potential locations. The grid of all potential DOAs is $\Theta=\left\{\tilde{\theta}_{1}, \tilde{\theta}_{2}, \ldots, \tilde{\theta}_{P}\right\}$. Here $P \gg K$. Then the steering vectors for each element of $\boldsymbol{\Theta}$ is $\widetilde{\mathbf{A}}=\left[\boldsymbol{\alpha}\left(\tilde{\theta}_{1}\right), \boldsymbol{\alpha}\left(\tilde{\theta}_{2}\right), \ldots, \boldsymbol{\alpha}\left(\tilde{\theta}_{P}\right)\right]$. Since $\boldsymbol{\Theta}$ is known, $\widetilde{\mathbf{A}}$ is also known. Expression (4) can be represented with the overcomplete basis matrix $\widetilde{\mathbf{A}}$ as

$$
\mathbf{e}_{\max }=\widetilde{\mathbf{A}} \boldsymbol{\beta}+\boldsymbol{\varepsilon}
$$

where $\boldsymbol{\beta}$ denotes the sparse signal vector that has only $K$ nonzero components. $\boldsymbol{\varepsilon}$ is the residual due to measurement noise and model errors. 
The simplest version of the sparse representation problem without noise is to find the count of nonzero entries $\boldsymbol{\beta}$, which is denoted by $\|\boldsymbol{\beta}\|_{0}$. Since the $l_{1}$ norm is not a good convex approximation of the $l_{0}$ norm. Although can't get the optimal weights, we can find the property of it is that the value of the weights is smaller when $\tilde{\theta}_{p}=\theta_{k}$, larger when $\tilde{\theta}_{p} \neq \theta_{k}$.

In view of the above analysis, our goal is to find a kind of weights that satisfy the property of the optimal weights. And apparently, the optimal array weights vector is closely related to the array steering vector of the true signal. For the steering vector of the virtual signal, the output power of the array will be reduced accordingly. Therefore the weights of $l_{1}$ norm is inversely proportional to the output power. The greater the signal powers, the smaller the corresponding weights. With the overcomplete basis matrix, we can obtain the weight is

$$
w_{i}=\frac{T}{\sum_{t}\left|\widetilde{\mathbf{A}}(i)^{H} \mathbf{e}_{t}\right|^{2}}
$$

where $t=1,2, \ldots, T$ is the index of received signal frame. We consider few frames to average the weight value, and the result is more accurate. Then the weight matrix is

$$
\mathbf{W}=\operatorname{diag}\left[w_{1}, w_{2}, \ldots, w_{P}\right]
$$

We can construct a following optimization problem using $l_{1}$ norm constraint

$$
\left\{\begin{array}{c}
\min \|\mathbf{W} \boldsymbol{\beta}\|_{1} \\
\text { s.t. }\left\|\mathbf{e}_{\max }-\widetilde{\mathbf{A}} \boldsymbol{\beta}\right\|_{2} \leq \eta
\end{array}\right.
$$

where $\|\cdot\|_{1}$ denotes $l_{1}$ norm, $\|\cdot\|_{2}$ denotes $l_{2}$ norm and $\eta$ is a regularization parameter. The optimization problem can be solved by an optimization package called CVX. The DOA estimation can be obtained at dominant peaks of the reconstructed signal power spectrum.

\section{Experimental Results}

In this section, we name the proposed algorithm that uses the eigenvector weight as EVW- $l_{1}$, and we compare the performance of MUSIC, $l_{1}$-SVD[7], NSW- $l_{1}$ [8]. In all experiments, we suppose that there is a ULA of 6 sensors with the spacing half of the signal wavelength. The angle range is evenly divided into 360 grids with $0.5^{\circ}$ sampling from $-90^{\circ}$ to $90^{\circ}$. The number of sampling snapshots is 100 . There are two far-field narrowband signals from $20^{\circ}$ and $50^{\circ}$ impinge on the array.

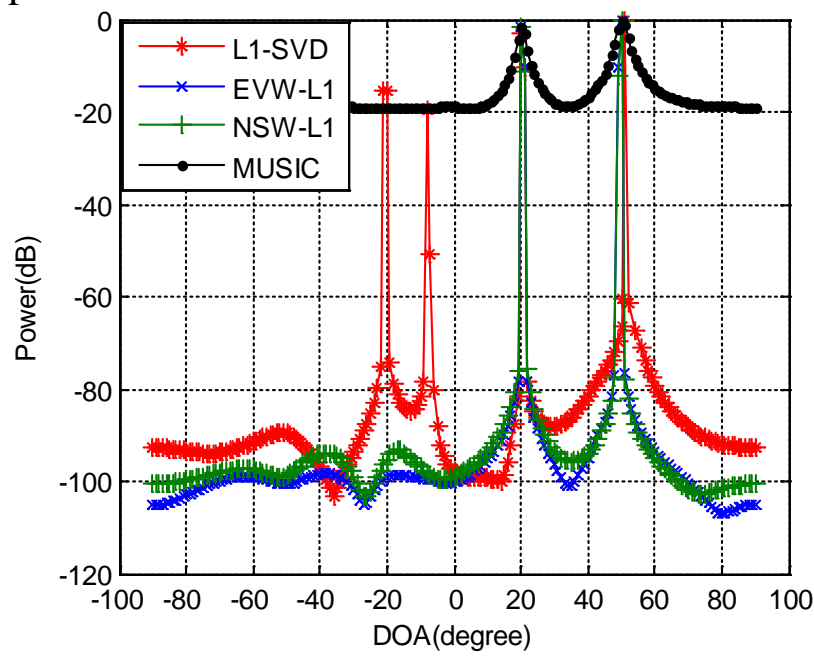

Fig 3 Spatial spectrum

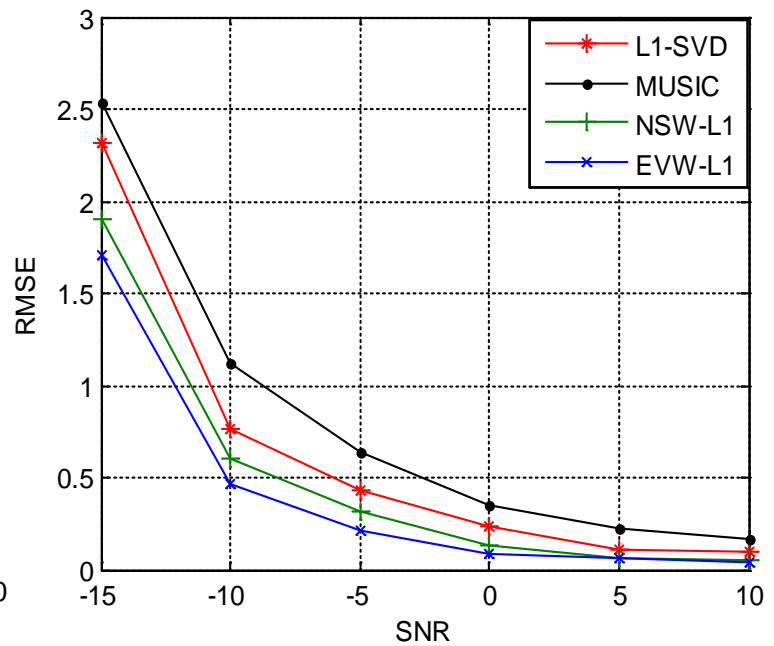

Fig 4 RMSE curve

First, The spatial spectrum obtained by the above algorithms for the case of 2dB SNR is shown in Fig 3. It can be seen the MUSIC algorithm spectrum peaks is not sharp enough which leads to large estimation error when the signal to noise is relatively low. The $l_{1}$-SVD algorithm will appear spurious peaks with low SNR. The proposed algorithm can resolve the two signals. 
Then we use root mean square error (RMSE) to evaluate the accuracy of the DOA estimation under varied SNR. Define the RMSE of the estimates from 50 trials as RMSE $=\sqrt{\frac{1}{50} \sum_{n=1}^{50} \sum_{i=1}^{2}\left(\hat{\theta}_{i n}-\theta_{i}\right)^{2}}$. In Fig 4, we can note that the RMSE of the proposed EVW- $l_{1}$ algorithm is lower than other mentioned algorithms.

\section{Conclusion}

In this paper, we present a weighted $l_{1}$ norm method for DOA estimation for vehicular applications. Since the signal is reconstructed based on the largest eigenvector of the covariance matrix, we don't need a prior knowledge of source number. Then the weight matrix of the largest eigenvector is used to enhance sparsity. Simulation results show that the proposed EVW- $l_{1}$ has higher estimation accuracy than the compared methods. And it is suitable for vehicular application.

\section{Acknowledgement}

In this paper, the research was sponsored by the National Natural Science Foundation of China (Project No. 61371115).

\section{References}

[1] Dar K, Bakhouya M, Gaber J, et al. Wireless communication technologies for ITS applications [J]. IEEE Communications Magazine, 2010, 48(5):156-162.

[2] Zhang J, Wang F Y, et al. Data-driven intelligent transportation systems: A survey [J]. IEEE Trans. on Intelligent Transportation Systems, 2011, 12(4): 1624-16339

[3] Sturm C, Zwick T, Wiesbeck W. An OFDM System Concept for Joint Radar and Communications Operations[C]// IEEE 69th Vehicular Technology Conference. 2009, 1 - 5

[4] Sturm C, Wiesbeck W. Waveform design and signal processing aspects for fusion of wireless communications and radar sensing [J]. Proceedings of the IEEE, 2011, 99(7): 1236-1259

[5] Krim H, Viberg M. Two decades of array signal processing research: the parametric approach [J]. IEEE Signal Processing Magazine, 1996, 13(4):67-94

[6] Donoho D L. Compressed Sensing[J]. IEEE Trans.inform.theory, 2006, 52(4):1289-1306.

[7] D. Malioutov, M. Cetin, A. S. Willsky. A sparse signal reconstruction perspective for source localization with sensor arrays [J]. Signal Processing IEEE Transactions on, 2005, 53(8):3010-3022

[8] Stoica P, Babu P, Li J. SPICE: A Sparse Covariance-Based Estimation Method for Array Processing [J]. Signal Processing IEEE Transactions on, 2011, 59(2):629-638

[9] Yin J, Chen T. Direction-of-Arrival Estimation Using a Sparse Representation of Array Covariance Vectors [J]. Signal Processing IEEE Transactions on, 2011, 59(9):4489-4493

[10] Zheng C, Li G, Wang X. An approach of DOA estimation using noise subspace weighted $l_{1}$ minimization[C]// 2011 IEEE International Conference, 2011:2856 - 2859

[11] X.Xu, X.Wei and Z.Ye, DOA Estimation Based on Sparse Signal Recovery Utilizing Weighted $l_{1}$-Norm Penalty [J]. IEEE Signal Processing Letters, 2012, 19(3):155-158 